\title{
Optimising Pharmacotherapy for Overactive Bladder: A Practical Guide to Safe \& Effective Prescribing of Anticholinergics (Including Combination Therapy with Beta-3 Agonists) In Patients with Overactive Bladder
}

\author{
Fawad ARIF, Arzu Yousuf, Mehwah Nadeem, Simon Fulford \\ Dept. of UROLOGY, James Cook University Hospital, Middlesbrough, United Kingdom.
}

*Corresponding Author: Fawad ARIF, Dept. of Urology, James Cook University Hospital, Marton road, Middlesbrough, TS4 3BW, United Kingdom.

\begin{abstract}
Objectives: Anticholinergics are often the mainstay of treatment for patients with overactive bladder. However, their efficacy is limited by their adverse effects especially in terms of cognitive impairment in the elderly. Recent evidence has confirmed that combination of anticholinergics with Beta-3adrenoceptor agonists is an effective, well tolerated \& safe option in these patients. This review provides a practical overview \& guide to safe \& effective prescription of anticholinergics as a monotherapy \& in combination with Beta 3-adrenoceptor agonists in terms of patient assessment/suitability, counselling, drug(s)selection/dosage \& patient monitoring (tolerability \& effectiveness), guiding a personalised approach to optimising pharmacotherapy in patients with overactive bladder.
\end{abstract}

Methods: The following 3 resources were utilized to write up a practical guide including formulation of a flow diagram encompassing patient \& drug factors:

1.Gratzke C, Chapple C et al. Efficacy \& safety of combination pharmacotherapy for patients with overactive bladder: A rapid evidence assessment. European Urology76(2019)767-779.

2.Fox C, Richardson K, Maidment ID, et al., Anticholinergic medication use and cognitive impairment in the older population: The Medical Research Council cognitive function and ageing study. J Am Geriatr Soc 2011; 59:1477-83.

3. Which anticholinergic drug for overactive bladder symptoms in adults Cochrane Systematic ReviewIntervention Version published:18 January 2012.

\section{Results:}

Flow-Diagram: for stepwise Patient evaluation \& Prescribing of Pharmacotherapy in patients with Overactive bladder.

Conclusions: Safe \& effective prescribing of pharmacotherapy can be achieved in a stepwise fashion, following focused urinary tract \& patient evaluation especially in terms of age, frailty, cognitive function \& anticholinergic burden. Most anticholinergics have comparable efficacy \& core side effect profile. Availability of Beta-3 Agonist therapy provides an additional treatment option, that can be utilised safely \& effectively, either on its own or in combination (with anticholinergics) to treat patients who are intolerant to standard anticholinergics or have poor symptom control. Patient counselling, use of standardised OAB symptom questionnaire \& focused review increases the likelihood of long-term success \& compliance.

Keywords: Overactive bladder, Anticholinergic, Antimuscarinics, Beta-3 Agonists, Pharmacotherapy

\section{Abbreviations:}

OAB- Overactive bladder

UI- Urge incontinence

UTI- Urinary tract infection

CNS- Central nervous system
ACB- Anticholinergic burden scale

MSU- Midstream urine

USKUB- Ultrasound kidney ureter bladder

eFI- Electronic fraility index 
Optimising Pharmacotherapy for Overactive Bladder: A Practical Guide to Safe \& Effective Prescribing of Anticholinergics (Including Combination Therapy with Beta-3 Agonists) In Patients with Overactive Bladder

\section{INTRODUCTION:}

Overactive bladder $(\mathrm{OAB})$ is characterised by urinary urgency, with or without incontinence, generally associated with increased frequency of micturition \& nocturia in the absence of a urinary tract infection (UTI) or other obvious pathology affecting the lower urinary tract ${ }^{4,5}$. Large scale population based surveys suggest a prevalence of $10 \%$ to $35 \%$ in the western world ${ }^{6}$, predominantly affecting elderly women ${ }^{7}$.

OAB bladder symptoms which are usually persistent \& long-term can be distressing \& have an adverse effect on the quality of life. These can affect daily physical activities such as shopping, travel, physical exercise in addition to sleep disturbances. Also, personal relationships especially in women can be affected due to fear of embarrassment. Women with urge urinary incontinence are more likely to be depressed ${ }^{8}$. Furthermore, OAB symptoms increase the risk of falls \& fractures, especially in the elderly. Extreme symptoms can lead to social isolation \& reclusion.

Patients with severe symptoms develop coping mechanisms such as fluid restriction, frequent voiding \& toilet mapping.

Historically pharmacotherapy with anticholinergics has been the mainstay of medical management in symptomatic patients with overactive bladder. However, their benefits $\&$ efficacy has been somewhat limited due to their well-known adverse effects especially as far as the potential for cognitive impairment in the aging population is concerned. The advent \& availability of Beta-3 agonists has changed the dynamics in the management of overactive bladder symptoms including combination therapy with standard antimuscuarinics.

There is paucity in the literature on the practical steps \& guides in patient evaluation \& assessment \& drug selection \& monitoring, as far as safe \& effective prescribing of oral pharmacotherapy is concerned in management of overactive bladder.

\section{MeTHODS}

The following 3 resources were utilized to write up a practical guide including formulation of a flow diagram encompassing patient \& drug factors:

1. Gratzke C, Chapple C et al. Efficacy \& safety of combination pharmacotherapy for patients with overactive bladder: A rapid evidence assessment. European Urology76(2019)767-779.

\section{Fox C, Richardson K, Maidment ID, et al}

Anticholinergic medication use and cognitive impairment in the older population: The Medical Research Council cognitive function and ageing study. J Am Geriatr Soc 2011; 59:1477-83.

3. Which anticholinergic drug for overactive bladder symptoms in adults Cochrane Systematic Review-Intervention Version published:18 January 2012.

\section{RESULTS}

\section{Flow-Diagram for Stepwise Patient Evaluation \& Prescribing of Pharmacotherapy in Patients with Overactive Bladder.}

Figure1. Flow Diagram $O A B$

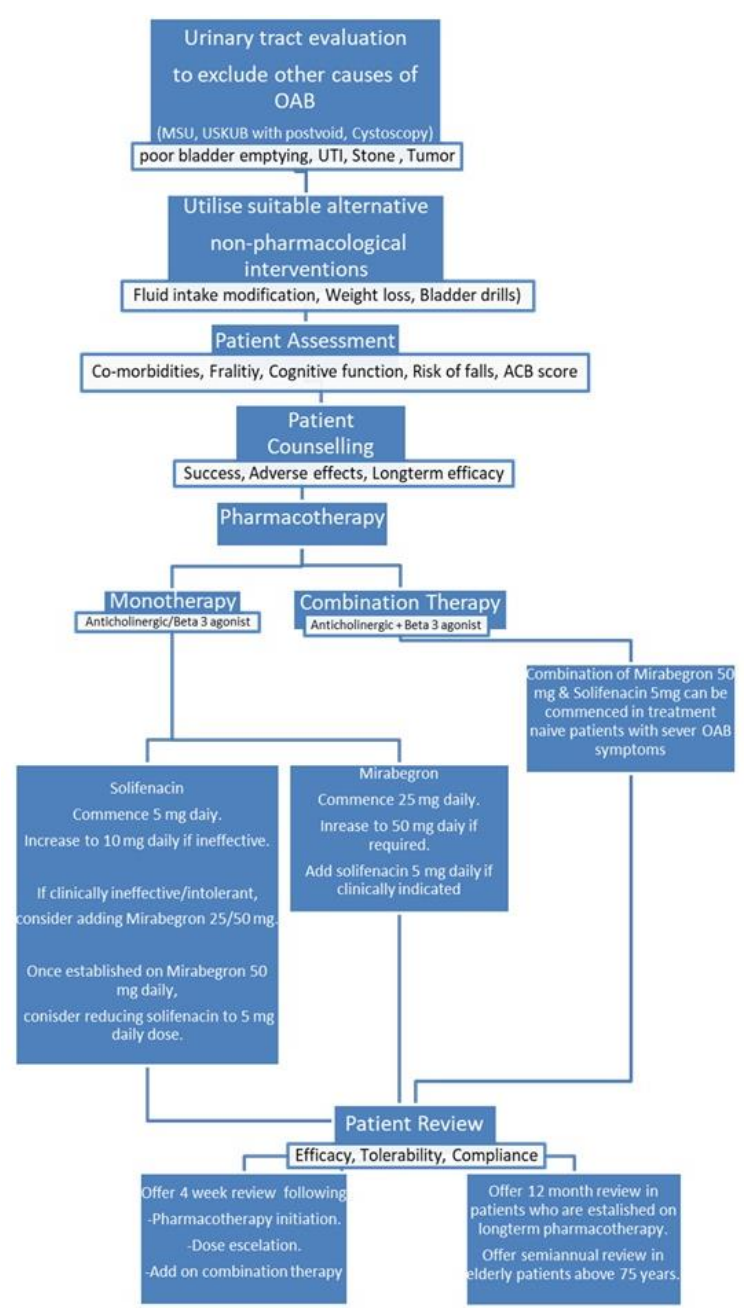

Figure Legend

Flow diagram depicting practical stepwise patient evaluation \& assessment \& drug(s) selection \& monitoring for optimal, safe \& 
Optimising Pharmacotherapy for Overactive Bladder: A Practical Guide to Safe \& Effective Prescribing of Anticholinergics (Including Combination Therapy with Beta-3 Agonists) In Patients with Overactive Bladder

affective pharmacological management of overactive bladder.

\section{DisCUSSION}

Traditionally anticholinergics (antimuscarinics) have been the first line pharmacotherapy to manage OAB. However their adverse effects especially with higher doses reduce their tolerability \& simultaneously risks increasing the anticholinergic burden, particularly in elderly patients who may already be on other anticholinergic medication ${ }^{9}$. This has led to development of alternate pharmacological agents such as Beta-3 adrenoceptor agonists (Mirabegron) \& Botulinum Toxin with different mechanisms of action, hence avoiding anticholinergic side effects.

\section{Combination Therapy}

Beta-3 adrenoceptor agonists such as Mirabegron $\&$ antimuscarinics have similar efficacy with the former having a more favourable tolerability profile $^{10}$. Evidence has shown that combination of the two is an effective \& well tolerated treatment option with long term clinically meaningful improvement in symptoms especially in adult patients with inadequate control of $\mathrm{OAB}$ symptoms on monotherapy as well as in treatment naïve patients ${ }^{3}$.

\section{Anticholinergic Adverse Events}

Antimuscarinics have poor tolerability \& compliance due to the anticholinergic effects such as dry mouth, constipation \& blurred vision $^{10}$. However anticholinergic medications are also associated with increased risk of cognitive impairment \& falls in patients over 65 years of age. Research has shown a link to increased risk of cognitive impairment \& mortality from anticholinergic drugs with the risk shown to be cumulative, based on the number of anticholinergics taken \& the strength of each drug's anticholinergic effect ${ }^{2}$.

\section{Anticholinergic Syndrome}

Dysfunction of the autonomic parasympathetic (cholinergic) nervous system can lead to systemic (peripheral) \& central nervous system (CNS) symptoms. Mild peripheral symptoms such as dry mouth, constipation \& blurred vision are common place \& may indicate that the medication is starting to have an effect. However severe systemic symptoms may manifest as photophobia, flushed \& dry skin, tachycardia, hypertension \& fever, in addition to frequently reduced gastrointestinal \& urinary motility. CNS symptoms include delirium, agitation, disorientation, visual hallucination \& seizures in extreme cases.

CNS symptoms can manifest in the absence of peripheral symptoms. Not all symptoms may manifest in patients with chronic anticholinergic toxicity when on occasions they could be wrongly attributed to another diagnosis.

\section{Anticholinergic Burden}

Numerous medications commonly prescribed in daily practice not thought of as classical anticholinergics, have significant anticholinergic effect. These commonly include antihistamines, tricyclic antidepressants, drugs for asthma \& COPD, cold preparations, anti-hypertensives, anti-emetics, anti-spasmodics, steroids, opioids, warfarin etc.

The anticholinergic burden scale-ACB (Table 1: ACB Score of commonly prescribed medication) lists the anticholinergic activity of commonly prescribed drugs on a scale of 1 (weak/possible anti-cholinergic effect) to 3 (definite anticholinergic effect) with a $70 \%$ risk of two or more adverse effects if the total ACB score is 3 or more (Table 2: ACB Scale \& risks).

Each definite anticholinergic (ACB score 2/3), increases the cognitive risk by $46 \%$ over 6 years 20.

Research evidence suggests ${ }^{2}$ that $20 \%$ of patients above 65 years of age who had an ACB score of four or more had died by the end of the two year study period compared with $7 \%$ of patients with an ACB score of zero. For every additional ACB point scored the risk of dying increased by $26 \%$ More importantly, the evidence showed this risk to be cumulative, based on number of anticholinergic drugs taken \& strength of each drug's anticholinergic effect-The ACB score ${ }^{2}$.

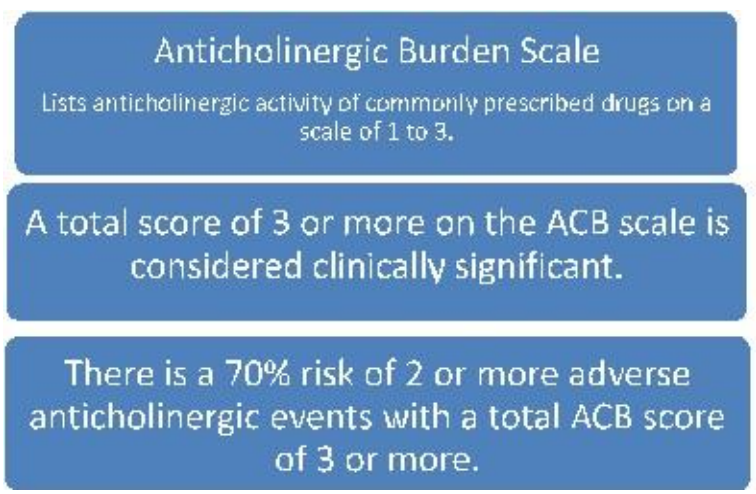


Optimising Pharmacotherapy for Overactive Bladder: A Practical Guide to Safe \& Effective Prescribing of Anticholinergics (Including Combination Therapy with Beta-3 Agonists) In Patients with Overactive Bladder

\begin{tabular}{|l|l|l|l|l|}
\hline \hline \multicolumn{5}{|c|}{ ACB Score of Commonly Prescribed Medication. } \\
\hline ACB Score 1 & ACB score 2 & ACB Score 3 \\
\hline Weak/possible anticholinergic effect. & \multicolumn{1}{l|}{ Definite anticholinergics. } \\
\hline Atenolol & Hydralazine & Amantadine & Amitryptyline & Oxybutynin \\
\hline Captopril & Haloperidol & Belladonaa & Atropine & Propenthaline \\
\hline Cimetidine & Hydrocortisone & Carbamezapine & Chlorphenamine & Solifenacin \\
\hline Codeine Phosphate & Isosorbide nitrate & Cyclobenzaprine & Chlorpromazine & Tolterodine \\
\hline Colchicine & Lopramide & Loxapine & Darifenacin & Trospium chloride. \\
\hline Digoxin & Loratidine & Nefopam & Fesoterodine & \\
\hline Diazepam & Morphine & Pimozide & Flavoxate & \\
\hline Dipyridamole & Cyclizine & & Hyoscyamine sulfate & \\
\hline Fentanyl & Predinisolone & & Imipramine & \\
\hline Furosemide & Warfarin Sodium & & Nortriptyline & \\
\hline
\end{tabular}

\section{Step Wise Practical Guide to Pharmacotherapy in Patients With OAB}

Figure1. Flow Diagram $O A B$

It is assumed that all suitable alternative nonpharmacological interventions such as fluid intake modification, weight loss, bladder drills would have been tried before initiating pharmacotherapy.

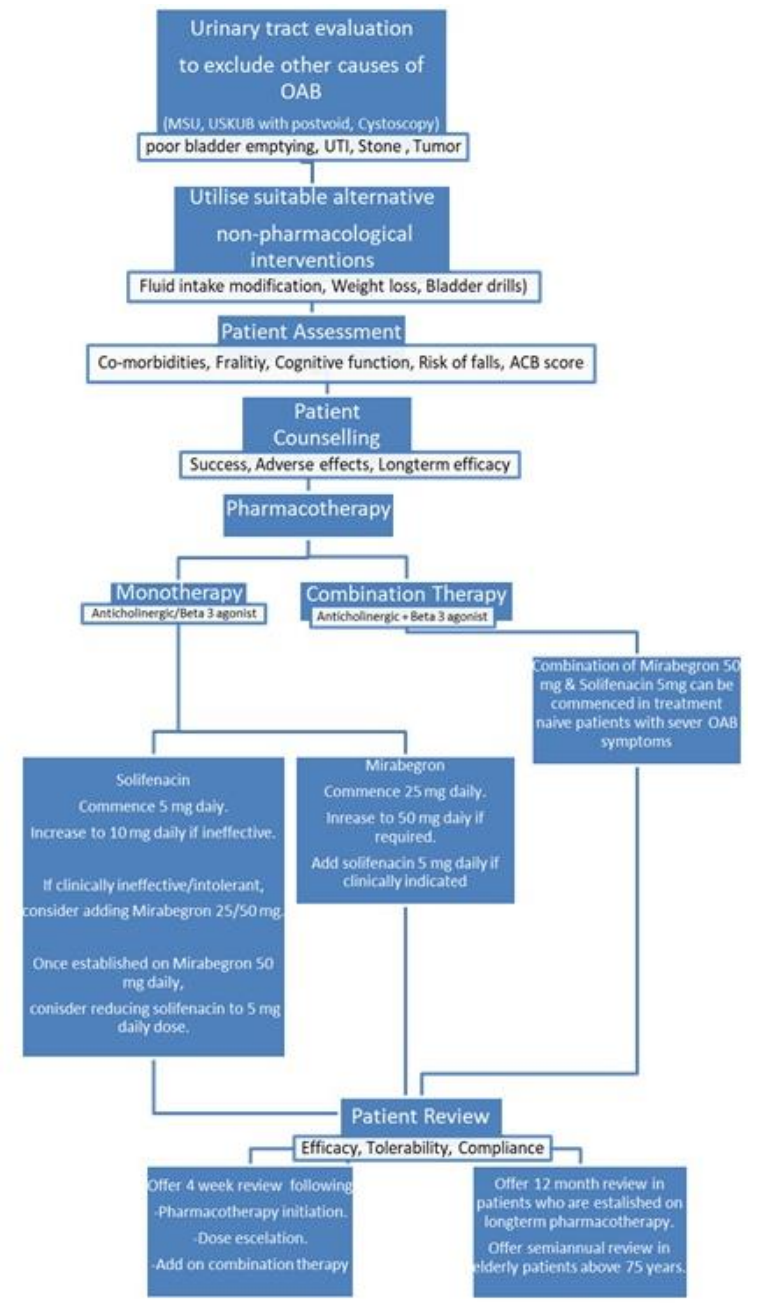

Evaluation of the urinary tract, including urine analysis, ultrasound kidney, ureter, bladder (USKUB) with post void \& flexible cystoscopy if indicated, is required to exclude other causes of OAB symptoms such as poor bladder emptying, UTI, stones, tumors etc.

\section{Patient Assessment}

It is important to assess patients in terms of suitability \& tolerance to anticholinergics.

\section{Frailty \& Age}

Assessment \& evaluation of the elderly (>65 years of age) \& frail population with complex comorbidities on multiple long term medication who are more susceptible to falls, immobility \& delirium, as a consequence of the side effects of the medication, is required prior to commencement of anticholinergic therapy.

\section{Cognitive Function:}

The clinician needs to take into account the patients cognitive function before initiating anticholinergic therapy. Patients at risk or suspected of having dementia require referral to specialist dementia diagnostic service. In high risk patients consider avoiding anticholinergics altogether \& consider suitable alternatives such as beta 3 adrenoceptor agonists.

\section{Risk of Falls}

A number of authoritative geriatric medicine sources, including findings from the health ABC study ${ }^{12}$ report that anticholinergic use may increase falls risk. Indeed, it is plausible that anticholinergic use could be related to falls as a result of their effect on the central nervous system (which may include ataxia, cognitive impairment, dizziness, and light-headedness) as well as other possible effects, such as mydriasis, 
Optimising Pharmacotherapy for Overactive Bladder: A Practical Guide to Safe \& Effective Prescribing of Anticholinergics (Including Combination Therapy with Beta-3 Agonists) In Patients with Overactive Bladder

causing a loss of accommodation and visual problems making elderly patients more vulnerable to loss of balance \& falls.

Anticholinergics may reduce sweating, leading to hyperthermia and fainting in hot environments as well.

\section{Frailty Index}

Since 2017, general practice (GP) surgeries in England are required to identify elderly patients above 65 years of age who are living with moderate to severe frailty by utilising the Electronic Frailty Index (eFI) which uses the existing information in the electronic primary healthcare records. The eFI measures frailty by accumulating a range of deficits including clinical signs (e.g.: tremors), symptoms (e.g.: blindness), disease, disability \& abnormal test values. It has been recommended by National Health Service England, that patients with severe ( $3 \%$ of over $65 \mathrm{~s}) \&$ moderate $(12 \%$ of over $65 \mathrm{~s})$ frailty, be considered for an annual medicine review \& risk falls assessment. This group is particularly vulnerable to anticholinergic therapy that should be considered cautiously.

\section{Assessment for Anticholinergic Burden (ACB Score)}

Remember to evaluate patient prescription for long term medications with significant anticholinergic activity. If ACB score is equal or greater than 3 , consider alternative therapy such as Mirabegron rather than commencing anticholinergic therapy due to higher risk of adverse events, especially in elderly patient with multiple comorbidities on multiple medication.

\section{Patient Counselling}

Counsel patients appropriately before starting anti cholinergic therapy. Inform them of the likelihood of success, adverse effects (including long term risk of cognitive decline) \& need to continue with medication in the long-term, as the beneficial effects may not be obvious for at least 4 weeks following commencement of medication as confirmed by the SYNERGY trial ${ }^{13} \&$ the likelihood of symptomatic improvement over time ${ }^{14}$.

\section{OAB Questionnaire:}

Use of a standardised $\mathrm{OAB}$ questionnaire encompassing symptom \& quality of life assessments such as International consultation on Incontinence questionnaire (ICIQ), recommended by British Association of
Urological Surgeons (BAUS) are not only helpful in initial evaluation of patients with overactive bladder but are also beneficial on subsequent reviews to monitor treatment progression \& impact on quality of life. OAB questionnaires help in counselling patients expectations \&/or satisfaction with treatment.

\section{Choosing Medicine for $\mathrm{OAB}$ :}

Pharmacotherapy management options for overactive bladder are three-fold including monotherapy in the form of anticholinergics or beta-3 adrenoceptor agonist therapy or a combination of the two. Use of anticholinergic monotherapy to treat patients with overactive bladder has been the standard of care for decades as far as pharmacotherapy in patients with OAB is concerned. However advent of beta-3 adrenoceptor agonist therapy has heralded a new era in management of these patients.

Recent European, American \& Canadian Urological Association guidelines recommend antimuscarinic or mirabegron monotherapy as a first or second line option for the management of $\mathrm{OAB} /$ Urge incontinence (UI) and combination therapy with mirabegron plus

antimuscarinics in patients whose symptoms are refractory to monotherapy. However National Institute for Health \& Care Excellence (NICE) guidelines $^{15}$ in the United Kingdom presently only recommend commencement of Mirabegron in patients, in whom antimuscarinic drugs are contraindicated, are clinically ineffective or have unacceptable side effects.

\section{Monotherapy:}

\section{Anticholinergics}

The Cochrane systemic review ${ }^{15}$ analysed various studies to assess which anticholinergic drugs to prescribe in adult symptomatic patients. The review found that there were various anticholinergics being prescribed for $\mathrm{OAB}$ patients, with the four most common ones being oxybutynin, tolterodine, solifenacin \& fesoterodine. Oxybutynin had similar efficacy compared to tolterodine but higher withdrawal rate due to adverse effects mainly a dry mouth especially with the immediate release preparation. Solifenacin had better efficacy \& less risk of dry mouth compared to tolterodine. Fesoterodine had better efficacy but poor tolerance due to adverse effects compared to tolterodine. A recent meta-analysis ${ }^{16}$ suggests 
Optimising Pharmacotherapy for Overactive Bladder: A Practical Guide to Safe \& Effective Prescribing of Anticholinergics (Including Combination Therapy with Beta-3 Agonists) In Patients with Overactive Bladder

that solifenacin \& tolterodine have similar efficacy \& incidence of dry mouth. However, tolterodine was noted to reduce the constipation rate, especially at 12 weeks.

Commencement with lowest dose of an Extended release anticholinergics is recommended rather than immediate release preparations (such as Oxybutynin) especially in elderly population above 65 years of age due increased risk of sudden deterioration in physical \& mental health 14. Solifenacin, trospium, fesoterodine \& Darfienacin have not shown to worsen cognitive impairment in short term studies ${ }^{11}$. However, do not offer flavoxate, propantheline or imipramine to treat UI or OAB in patients above 65 years of age $^{14}$.

\section{Beta 3 Adrenoceptor Agonists}

Mirabegron (Betigma) is a potent \& selective beta-3 adrenoceptor agonist which relaxes the detrusor smooth muscle during the storage phase of the urinary bladder fill-void cycle, hence increasing the bladder capacity \& reducing the symptoms of an overactive bladder. As previously mentioned, Mirabegron has a more favourable tolerability profile compared to anticholinergics with similar/comparable clinical efficacy ${ }^{10}$.

\section{Combination Therapy}

Recent rapid evidence assessment ${ }^{3}$ of efficacy \& safety of combination pharmacotherapy which included evaluation of phase II (SYMPHONY) $\&$ phase III (SYNERGY/SYNERGYII/BESIDE) trials showed that a combination of Mirabegron $50 \mathrm{mg} \&$ Solifenacin $5 \mathrm{mg}$ daily, provides effective \& well tolerated treatment option in adults with inadequate control of $\mathrm{OAB}$ symptoms on monotherapy as well as in treatment naïve patients with severe symptoms. The optimal dose of solifenacin in combination with beta-3 agonist was deemed to be $5 \mathrm{mg}$ rather than $10 \mathrm{mg}$, as the latter had a marginally increased efficacy at the expense of higher antimuscarinic side effects.

\section{Reviewing Medication}

Patients should be assessed for efficacy, tolerability \& compliance after 4 weeks of commencement of monotherapy (antimuscarinic/mirabegron) either in person or via a telephone consultation. Similarly, all patients should be reviewed in 4 weeks following dose escalation or add on therapy. Patients who are established on long-term medication with good symptomatic control for overactive bladder or urge incontinence should have a yearly review. Offer a semi-annual review if they are above 75 years of age $^{14}$ due to higher risk of adverse events.

Safe \& effective prescribing of pharmacotherapy can be achieved in a stepwise fashion, following focused urinary tract \& patient evaluation especially in terms of age, frailty, cognitive function \& anticholinergic burden. Most anticholinergics have comparable efficacy \& core side effect profile ${ }^{19}$. Availability of beta-3 agonist therapy provides an additional treatment option, that can be utilised safely \& effectively, either on its own or in combination (with anticholinergics) to treat patients who are intolerant to standard anticholinergics or have poor symptom control. Patient counselling, use of standardised $\mathrm{OAB}$ symptom questionnaire \& focused review increases the likelihood of longterm success \& compliance.

\section{Conclusions:}

Safe \& effective prescribing of pharmacotherapy can be achieved in a stepwise fashion, following focused urinary tract \& patient evaluation especially in terms of age, frailty, cognitive function \& anticholinergic burden. Most anticholinergics have comparable efficacy \& core side effect profile. Availability of Beta-3 Agonist therapy provides an additional treatment option, that can be utilised safely \& effectively, either on its own or in combination (with anticholinergics) to treat patients who are intolerant to standard anticholinergics or have poor symptom control. Patient counselling, use of standardised $\mathrm{OAB}$ symptom questionnaire \& focused review increases the likelihood of longterm success \& compliance.

\section{REFERENCES}

[1] Herbison P, Hay-Smith J, Ellis G, Moore K. Effectiveness of anticholinergic drugs compared with placebo in the treatment of overactive bladder: systematic review. BMJ2003; 326:8414.

[2] Fox C, Richardson K, Maidment ID, et al. Anticholinergic medication use and cognitive impairment in the older population: the medical research council cognitive function and ageing study. J Am Geriatr Soc 2011; 59:1477-83.

[3] Gratzke C, Chapple C et al. Efficacy \& safety of combination pharmacotherapy for patients with overactive bladder: A rapid evidence 
Optimising Pharmacotherapy for Overactive Bladder: A Practical Guide to Safe \& Effective Prescribing of Anticholinergics (Including Combination Therapy with Beta-3 Agonists) In Patients with Overactive Bladder

assessment. European Urology 76(2019) 767779.

[4] Drake MJ. Do we need a new definition of the overactive bladder Syndrome? ICI-RS 2013. Neurourol Urodyn 2014; 33:622-4.

[5] Abrams P, Cardozo L, Fall M, et al. The standardisation of terminology in lower urinary tract function: report from the standardization Sub-committee of the International Continence Society. Urology 2003; 61:37-49.

[6] Eapen RS, Radomski SB. Review of the epidemiology of overactive Bladder. Res Rep Urol 2016; 8:71-6.

[7] Irwin DE, Kopp ZS, Agatep B, Milsom I, Abrams P. Worldwide Prevalence estimates of lower urinary tract symptoms, overactive bladder, urinary incontinence and bladder outlet obstruction. BJU Int 2011; 108:1132-8.

[8] Zorn BH, Montgomery H, Pieper K, Gray M, Steers WD. Urinary incontinence and depression. J Urol1999; 162:82-4

[9] Yoshida M, Kato D, Nishimura T, Van Schyndle J, Uno S, Kimura T. Anticholinergic burden in the Japanese elderly population: use of antimuscarinic medications for overactive bladder patients. Int J Urol 2018; 25:855-62.

[10] Deeks ED. Mirabegron: a review in overactive bladder syndrome. Drugs 2018; 78:833-44.

[11] Kerdraon J, Robain G, Jeandel C et al. Groupe de recherche appliquée à la pelvi-périnéologie de la personne âgée (Impact on cognitive function of anticholinergic drugs used for the treatment of overactive bladder in the elderly.). Prog Urol. 2014 Sep;24(11):672-81. doi: 10.1016/j.purol.2014.06.003. Epub 2014 Jun 30. [Article in French]

[12] Zachary A. Marcum, Subashan Perera, Joshua M. Thorpe et al: . Anticholinergic Use and Recurrent Falls in Community-Dwelling Older Adults: Findings From the Health ABC Study,
USA. Ann Pharmacother. 2015 Nov; 49(11): 1214-1221.

[13] Herschorn S, Chapple CR, Abrams $\mathrm{P}$ et al: Efficacy and safety of combinations of mirabegron and solifenacin compared with monotherapy and placebo in patients with overactive bladder (SYNERGY study). BJU Int. 2017 Oct;120(4):562-575. doi: 10.1111/bju.13882. Epub 2017 Jun 8.

[14] Urinary incontinence and pelvic organ prolapse in women: management NICE guideline [NG123]

[15] Which anticholinergic drug for overactive bladder symptoms in adults Cochrane Systematic Review - Intervention Version published: 18 January 2012

[16] Wang H.-T, Xia M.Comparisons of Therapeutic Efficacy and Safety of Solifenacin versus Tolterodine in Patients with Overactive Bladder: A Meta-Analysis. Urol Int 2019; 103:187-194

[17] Mirabegron for treating symptoms of overactive bladder Technology appraisal guidance [TA290] Published date: 26 June 2013

[18] Abrams P, Kelleher C, Staskin D et al: Combination treatment with mirabegron and solifenacin in patients with overactive bladder: efficacy and safety results from a randomised, double-blind, dose-ranging, phase 2 study (Symphony). Eur Urol. 2015 Mar;67(3):577-88. doi: 10.1016/j.eururo.2014.02.012. Epub 2014 Feb 19.

[19] Jayarajan J, Sidney R: Pharmacotherapy of overactive bladder in adults: a review of efficacy, tolerability, and quality of life Res Rep Urol. 2014; 6: 1-16.

[20] Campbell N, Boustani M, Lane K, et al. Use of anticholinergics and the risk of cognitive impairment in an African American population. Neurology. 2010; 75:152-159.

Citation: Fawad ARIF, Arzu Yousuf, Mehwah Nadeem, Simon Fulford. Optimising Pharmacotherapy for Overactive Bladder: A Practical Guide to Safe \& Effective Prescribing of Anticholinergics (Including Combination Therapy with Beta-3 Agonists) In Patients with Overactive Bladder. ARC Journal of Urology. 2021; 6(1):1-7. DOI: https://doi.org/ 10.20431/2456-060X.060102.

Copyright: (C) 2021 Authors. This is an open-access article distributed under the terms of the Creative Commons Attribution License, which permits unrestricted use, distribution, and reproduction in any medium, provided the original author and source are credited. 